\title{
EP-160
}

\section{Laparoscopic pancreaticoduodenectomy for periampullary tumors: A contraindication for octogenarians?}

\author{
$\underline{\text { Ji Su KIM }}{ }^{1,2}$, Munseok $\mathrm{CHOl}^{1,2}$, Sung Hyeon $\mathrm{KIM}^{1,2}$, Sung Hoon $\mathrm{CHOl}^{3}$, Chang Moo KANG ${ }^{* 1,2}$ \\ 'Department of Hepatobiliary and Pancreatic Surgery, Yonsei University College of Medicine, Seoul, Korea \\ ${ }^{2}$ Department of Pancreatobiliary cancer center, Yonsei Cancer Center, Severance Hospital, Seoul, Korea \\ ${ }^{3}$ Department of Hepatobiliary and Pancreatic Surgery, CHA Bundang Medical Center, Seongnam, Korea
}

Introduction: With continued technical advances in surgical instruments and the growing surgical expertise, many laparoscopic pancreaticoduodenectomies (LPDs) have been safely performed with favorable outcomes, and this approach is being used more frequently. Interest in treatments for elderly patients has increased with life expectancy. We investigated safety and feasibility of LPD in ontogenarians.

Methods: From September 2005 to February 2020, resectable/borderline resectable periampullary tumors (PATs) were diagnosed in 71 octogenarians at Sincheon Severance Hospital and CHA Bundang Medical Center. Patients were divided into two groups: those who underwent surgery $(\mathrm{N}=38)$ and those who did not $(\mathrm{N}=33)$. The surgery group was further divided into two groups: those who underwent open pancreaticoduodenectomies (PDs) (OPDs) $(\mathrm{N}=19)$, and those who underwent LPDs $(\mathrm{N}=19)$. Perioperative outcomes, including long-term survival, were retrospectively compared.

Results: There was no significant difference in age, sex, comorbidities, diagnosis, and chemo-radiotherapy between the surgery and non-surgery groups. The surgery group had a better survival rate than the non-surgery group $(p<0.05)$. Baseline characteristics and postoperative outcomes were not significantly different between the OPD and LPD groups. Only three patients in the OPD group and two patients in the LPD group had a biochemical leak $(p>0.05)$. There was no significant difference between the OPD and LPD groups in overall survival and disease-free survival $(p=0.816, p=0.446$, respectively).

Conclusions: PD can improve survival outcome in octogenarian patients with PATs. Even LPD can be carefully performed with comparable oncologic outcomes in well-selected octogenarian patients. 